\title{
Swing-up Control for $n$-Link Planar Robot with Single Passive Joint Using the Notion of Virtual Composite Links
}

\author{
Xin Xin, Jin-Hua She, and Taiga Yamasaki
}

\begin{abstract}
In this paper, we concern a swing-up control problem for an $n$-link revolute planar robot with any one of the joint being a passive joint. The goal of this study is to design and analyze a swing-up controller that can bring the robot into any arbitrarily small neighborhood of the upright equilibrium point with all links in the upright position. To achieve this challenging objective while preventing the robot from becoming stuck at an undesired closed-loop equilibrium point, first, we address how to iteratively devise two series of virtual composite links separated by the passive joint to be used for designing a coordinate transformation on the angles of all active joints. Second, we devise an energy based swing-up controller that uses a new Lyapunov function based on that transformation. Third, we analyze the global motion of the robot under the controller and establish conditions on control parameters that ensure attainment of the swing-up control objective. The results obtained here unify some previous results for the Pendubot, the Acrobot, and three-link robots with a passive first joint. Finally, we validate the theoretical results via a numerical simulation investigation to a 4-link robot with a passive joint.
\end{abstract}

\section{INTRODUCTION}

The last two decades have witnessed considerable progress in studying underactuated robots, which possess fewer actuators than degrees of freedom [1], [3], [11]. A robot may be underactuated as a result of intentional design to reduce weight and cost, or as a result of actuator failure. Since underactuated robots usually have nonholonomic secondorder constraints, the control problems are challenging [6], [9].

There are many researches on two-degree-of-freedom (2DOF) pendulum type robots, e.g., [2], [5], [8], [12]. The energy based control approach, which has been developed in the seminal works of [4], [7], [11], has been shown theoretically effective for solving the swing-up control problem for the Pendubot in [4], [7] and the Acrobot in [13].

A swing-up control problem was studied in [14] for a 3link planar robot in a vertical plane with a passive first joint. For this robot, [14] showed that, unlike 2-DOF underactuated robots ([4], [7], [13]), it is difficult to analyze the motion when it is governed by a swing-up controller employing a conventional Lyapunov function, which contains the energy of the robot, and the angles and angular velocities of the two active joints. To overcome this difficulty, [14] treated the links 2 and 3 as a virtual composite link and proposed a coordinate transformation on the angles of two active joints.

This work was supported in part by a Grant-in-aid Scientific Research (C) under grant no. 19560452 .

X. Xin and T. Yamasaki are with Faculty of Computer Science and System Engineering, Okayama Prefectural University, 111 Kuboki, Soja, Okayama 719-1197, Japan \{xxin, taiga\}@c.oka-pu.ac.jp

J.-H. She is with the School of Computer Science, Tokyo University of Technology, 1404-1 Katakura, Hachioji, Tokyo, 192-0982, Japan she@cc.teu.ac.jp
In this paper, we investigate how to extend the design and analysis of the swing-up control for the 3-link robot in [14] to a general $n$-link planar robot with any one of the joint being a passive joint. Note that some preliminary results are presented in [15] for the case of a passive first joint. To guarantee that the robot can enter the basin of attraction of any stabilizing controller for the upright equilibrium point, where all links are in the upright position, we aimed to design and analyze a swing-up controller that drives the robot from any initial state into any arbitrarily small neighborhood of the upright equilibrium point.

To achieve this challenging objective, we need to prevent an $n$-link robot from becoming stuck in an undesired closedloop equilibrium configuration. Our idea for clarifying the structures of the closed-loop equilibrium configurations is to iteratively study $n-1$ robots of the Acrobot type (that is, two links with a passive first joint) and/or the Pendubot (that is, two links with a passive second joint) rather than directly studying an $n$-link robot. First, we address the problem of how to iteratively devise two series of virtual composite links separated by the passive joint to be used for designing a coordinate transformation on the angles of all active joints. Second, we construct a Lyapunov function based on the transformation and use it to design a swing-up controller. Third, we analyze the global motion of the robot under the controller and establish conditions on control parameters that ensure attainment of the swing-up control objective. In fact, by using the virtual composite links, we succeed in revealing a relationship between the closed-loop equilibrium points and a control parameter. The results obtained unify some previous results for the Pendubot, the Acrobot, and 3-link robots with a passive first joint. Finally, we verify the theoretical results via simulation for a 4-link robot with a passive joint.

\section{PRELIMINARIES AND PROBLEM FORMULATION}

\section{A. Model of n-Link Robot with Single Passive Joint}

Consider an $n$-link revolute robot with the $d$ th $(1 \leq d \leq n)$ joint being passive shown in Fig. 1 . For the $i$ th $(i=1, \ldots, n)$ link, $m_{i}$ is its mass, $l_{i}$ is its length, $l_{c i}$ is the distance from joint $i$ to its center of mass (COM), and $J_{i}$ is the inertia moment around its COM.

Let $q=\left[\begin{array}{cccc}q_{1}, & q_{2}, & \ldots, & q_{n}\end{array}\right]^{\mathrm{T}}$ be the vector of the angles of all the joints in generalized coordinates. In this paper, the angle of the passive joint, $q_{d}$, is dealt with in $\mathbb{S}$, which denotes a unit circle, while the vector of the angles of all the ac-

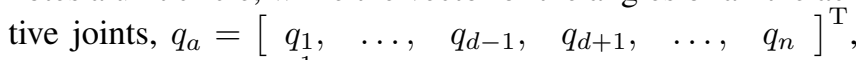
is dealt with in $\mathbb{R}^{n-1}$.

The motion equation of the robot is

$$
M(q) \ddot{q}+H(q, \dot{q})+G(q)=B \tau,
$$




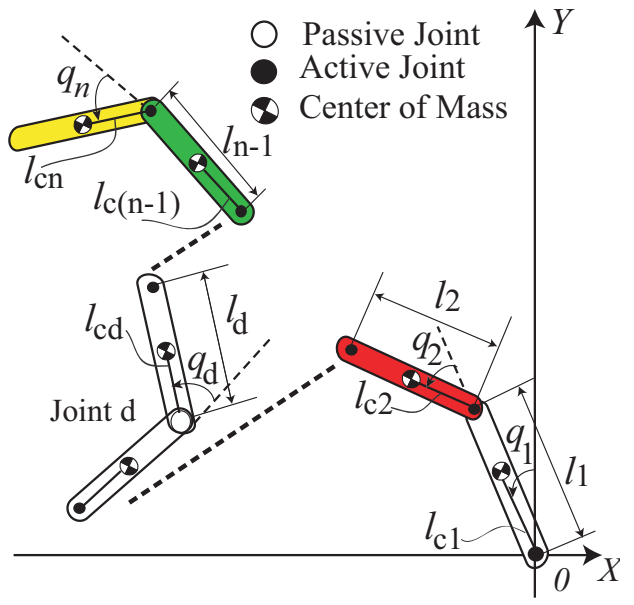

Fig. 1. An $n$-link robot with a passive $d$ th joint.

where $M(q) \in \mathbb{R}^{n \times n}$ is a symmetric positive definite inertia matrix, $H(q, \dot{q}) \in \mathbb{R}^{n}$ contains the Coriolis and centrifugal terms, $G(q) \in \mathbb{R}^{n}$ contains the gravitational terms, and $B$ is the input matrix of

$$
B=\left[\begin{array}{cc}
I_{d-1} & 0 \\
0 & 0 \\
0 & I_{n-d}
\end{array}\right] \in \mathbb{R}^{n \times(n-1)}
$$

with $I_{i}$ being an identity matrix of size $i$, and $\tau=$

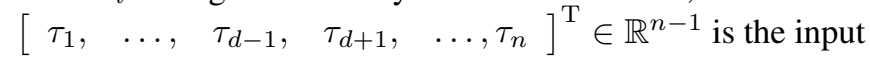
torque vector produced by $n-1$ actuators at active joints 1 , $\ldots, d-1, d+1, \ldots$, and $n$.

The energy of the robot is expressed as

$$
E(q, \dot{q})=\frac{1}{2} \dot{q}^{\mathrm{T}} M(q) \dot{q}+P(q),
$$

where $P(q)$ is the potential energy and is defined as

$$
P(q)=\sum_{i=1}^{n} m_{i} g Y_{G i}=\sum_{i=1}^{n} \beta_{i} \cos \sum_{j=1}^{i} q_{j},
$$

where $g$ is the acceleration of gravity, $Y_{G i}$ is the $Y$-axis coordinate of the COM of link $i$, and

$$
\beta_{i}=m_{i} l_{c i} g+\left(\sum_{j=i+1}^{n} m_{j}\right) l_{i} g, \quad \text { for } i=1, \ldots, n .
$$

Let $G_{i}(q)$ be the $i$ th element of $G(q)$. We have

$$
G_{i}(q)=\frac{\partial P}{\partial q_{i}}=-\sum_{k=i}^{n} \beta_{k} \sin \sum_{j=1}^{k} q_{j} .
$$

\section{B. Problem Formulation}

Consider the following upright equilibrium point:

$$
q=0, \quad \dot{q}=0 .
$$

For $E(q, \dot{q}), \dot{q}_{a}$, and $q_{a}$, if we can design $\tau$ such that

$$
\lim _{t \rightarrow \infty} E(q, \dot{q})=E_{r}, \quad \lim _{t \rightarrow \infty} \dot{q}_{a}=0, \quad \lim _{t \rightarrow \infty} q_{a}=0,
$$

where $E_{r}=\sum_{i=1}^{n} \beta_{i}$ is the energy of the robot at the upright equilibrium point, then from the analysis of Case 1 in Section
$\mathrm{V}$, we know that the robot can be swung up to any arbitrarily small neighborhood of the upright equilibrium point.

A Lyapunov function candidate for designing such $\tau$ is

$$
V_{A}=\frac{1}{2}\left(E-E_{r}\right)^{2}+\frac{1}{2} k_{D} \dot{q}_{a}^{\mathrm{T}} \dot{q}_{a}+\frac{1}{2} k_{P} q_{a}^{\mathrm{T}} q_{a},
$$

where scalars $k_{D}>0$ and $k_{P}>0$ are control parameters. However, similar to the discussion in [14], under the controller designed via such $V_{A}$. we find that it is difficult to determine the relationship between the control parameter $k_{P}$ and the closed-loop equilibrium configurations for completing the analysis of resulting closed-loop behavior. Thus, we shall propose a coordinate transformation on $q_{a}$ using the notion of virtual composite links for devising a different Lyapunov function.

\section{VIRTUAL COMPOSITE LINKS AND COORDINATE TRANSFORMATION}

\section{A. Virtual Composite Links}

We devise two series of virtual composite links (VCLs) separated by the passive joint $d$ as follows:

Series a: VCL $1, \ldots$, VCL $d-1$

For $1 \leq i \leq d-1$, we define VCL $i$ as a composite link of links 1 to $i$;

Series b: VCL $d+1, \ldots$ VCL $n$

For $d+1 \leq i \leq n$, we define VCL $i$ as a composite link of links $i$ to $n$.

We provide Tables I and II to describe the relationship between link $i$ and VCL $i$, where $1(0)$ denotes that an actual link is (not) a part of a VCL. Note that Series a (or b) is an empty set if $d=1$ (or $d=n$ ).

TABLE I

VCLS OF SERIES A

\begin{tabular}{lccccc}
\hline & link 1 & link 2 & $\ldots$ & link $d-2$ & link $d-1$ \\
\hline VCL 1 & 1 & 1 & $\ldots$ & 1 & 1 \\
\hline VCL 2 & 0 & 1 & $\ldots$ & 1 & 1 \\
\hline$\vdots$ & $\vdots$ & $\vdots$ & $\vdots$ & $\vdots$ & $\vdots$ \\
\hline VCL $d-2$ & 0 & 0 & $\ldots$ & 1 & 1 \\
\hline VCL $d-1$ & 0 & 0 & $\ldots$ & 0 & 1 \\
\hline
\end{tabular}

TABLE II

VCLS OF SERIES B

\begin{tabular}{lccccc}
\hline & link $d+1$ & link $d+2$ & $\ldots$ & link $n-1$ & link $n$ \\
\hline $\operatorname{VCL} d+1$ & 1 & 1 & $\ldots$ & 1 & 1 \\
\hline$\vdots$ & $\vdots$ & $\vdots$ & $\vdots$ & $\vdots$ & $\vdots$ \\
\hline $\operatorname{VCL} n-1$ & 0 & 0 & $\ldots$ & 1 & 1 \\
\hline $\operatorname{VCL} n$ & 0 & 0 & $\ldots$ & 0 & 1 \\
\hline
\end{tabular}

Although defining VCL $d-1$ and VCL $n$ as links $d-1$ and $n$ respectively somewhat abuses the idea of "virtual", such a definition allows us to iteratively construct two series of VCLs in a backward way (Fig. 2). In fact, for Series a, it starts from VCL $d-1$ being link $d-1$, and for $1 \leq i \leq d-2$, we see that VCL $i$ is a composite link of link $i$ and $V C L i+1$. For Series b, it starts from VCL $n$ being link $n$, and for $d+1 \leq i \leq n-1$, again we see that VCL $i$ as a composite link 
of link $i$ and $V C L i+1$. This iterative relationship facilitates the expression of the results in this paper in a concise way.

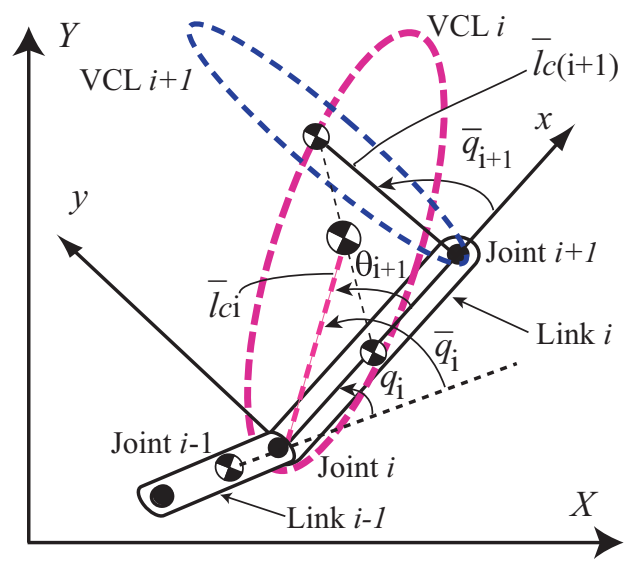

Fig. 2. Links $i-1$ and $i$, VCLs $i$ and $i+1$.

\section{B. Coordinate Transformation}

For VCL $i$ in Fig. 2, we define the following terms.

$\bar{q}_{i}$ : the angle of VCL $i$ with respect to link $i-1$; where for $i=1, \bar{q}_{1}$ is the angle with respect to the horizontal. $\theta_{i+1}$ : the angle of VCL $i$ with respect to link $i$.

$\bar{l}_{c i}$ : the distance between joint $i$ and the COM of VCL $i$. Moreover, when link $i$ and VCL $i+1$ are stretched out in a straight line $\left(\bar{q}_{i+1}=0\right)$, it is reasonable to define

$$
\theta_{i+1}=0, \text { when } \bar{q}_{i+1}=0 .
$$

Since Series a and $\mathrm{b}$ are separated by the passive joint $d$, we define $\bar{q}_{a}$ being the generalized coordinate vector related to the VCLs with the following decomposition:

$$
\bar{q}_{a}=\left[\begin{array}{c}
\bar{q}_{a a} \\
\bar{q}_{a b}
\end{array}\right], \quad\left\{\begin{array}{l}
\bar{q}_{a a}=\left[\begin{array}{lll}
\bar{q}_{1}, & \ldots, & \bar{q}_{d-1}
\end{array}\right]^{\mathrm{T}}, \\
\bar{q}_{a b}=\left[\begin{array}{lll}
\bar{q}_{d+1}, & \ldots, & \bar{q}_{n}
\end{array}\right]^{\mathrm{T}} .
\end{array}\right.
$$

Accordingly, we decompose $q_{a}$ as follows:

$$
q_{a}=\left[\begin{array}{l}
q_{a a} \\
q_{a b}
\end{array}\right], \quad\left\{\begin{array}{l}
q_{a a}=\left[\begin{array}{lll}
q_{1}, & \ldots, & q_{d-1}
\end{array}\right]^{\mathrm{T}} . \\
q_{a b}=\left[\begin{array}{lll}
q_{d+1}, & \ldots, & q_{n}
\end{array}\right]^{\mathrm{T}} .
\end{array}\right.
$$

We now determine the transformation $T: \mathbb{R}^{n-1} \rightarrow \mathbb{R}^{n-1}$ of $q_{a}$ to $\overline{q_{a}}$; that is,

$$
\bar{q}_{a}=T\left(q_{a}\right) .
$$

First, from Fig. 2, since $q_{i}$ is the angle of link $i$ with respect to link $i-1$, from the definitions of $\bar{q}_{i}$ and $\theta_{i+1}$, we obtain

$$
\overline{q_{i}}=q_{i}+\theta_{i+1}, \text { for } i \in \mathbb{N}_{d}, \text { with } \theta_{d}=\theta_{n+1}=0,
$$

where

$$
\mathbb{N}_{d}=\{i: 1 \leq i \leq d-1, \text { or, } d+1 \leq i \leq n\} .
$$

Thus, as to $\bar{q}_{a}$ and $q_{a}$, owing to (14) and (10), we have

$$
\bar{q}_{a}=0 \Longleftrightarrow q_{a}=0 .
$$

Next, we have the following observation: for $1 \leq i \leq d-1$, it follows from Table I that each $\theta_{i+1}$ is a function of $q_{i+1}$, $\ldots$, and $q_{d-1}$; while for $d+1 \leq i \leq n$, if follows from Table II that $\theta_{i+1}$ is a function of $q_{i+1}, \ldots$, and $q_{n}$. Therefore,

$$
\left\{\begin{array}{l}
\bar{q}_{i}=q_{i}+\theta_{i+1}\left(q_{i+1}, \ldots, q_{d-1}\right), \quad 1 \leq i \leq d-1, \\
\bar{q}_{i}=q_{i}+\theta_{i+1}\left(q_{i+1}, \ldots, q_{n}\right), \quad d+1 \leq i \leq n .
\end{array}\right.
$$

Since it is not easy to derive $\theta_{i+1}$ directly, in what follows, we express $\theta_{i+1}$ by using the fact that VCL $i$ is a composite link of link $i$ and $V C L i+1$.

To this end, as shown in Fig. 2, we use a Cartesian coordinate system $\left(x_{i}, y_{i}\right)$ with its origin at joint $i$ and its $x$-axis lying on link $i$. Since in these coordinates the coordinates of COMs of link $i$ and VCL $i+1$ are $\left(l_{c i}, 0\right)$ and $\left(l_{i}+\bar{l}_{c(i+1)} \cos \bar{q}_{i+1}, \bar{l}_{c(i+1)} \sin \bar{q}_{i+1}\right)$, respectively, letting $\left(x_{c i}, y_{c i}\right)$ be the coordinates of the COM of VCL $i$ yields

$$
\left(x_{c i}, y_{c i}\right)=\frac{\left(\beta_{i}+\bar{\beta}_{i+1} \cos \bar{q}_{i+1}, \bar{\beta}_{i+1} \sin \bar{q}_{i+1}\right)}{\bar{m}_{i} g},
$$

where

$$
\bar{\beta}_{i}=\bar{m}_{i} \bar{l}_{c i} g,
$$

where $\bar{m}_{i}=\sum_{j=i}^{n} m_{j}$ is the mass of VCL $i$.

Next, from (18), we obtain

$$
\left\{\begin{aligned}
\sin \theta_{i+1} & =\frac{y_{c i}}{\bar{l}_{c i}}=\frac{\bar{\beta}_{i+1} \sin \bar{q}_{i+1}}{\bar{\beta}_{i}}, \\
\cos \theta_{i+1} & =\frac{x_{c i}}{\bar{l}_{c i}}=\frac{\beta_{i}+\bar{\beta}_{i+1} \cos \bar{q}_{i+1}}{\bar{\beta}_{i}} .
\end{aligned}\right.
$$

Since $\theta_{i+1}$ is dealt with in $\mathbb{R}$ rather than $\mathbb{S}$, we cannot determine $\theta_{i+1}$ just from the values of its sine and cosine in (20). In fact, $\theta_{i+1}$ must also satisfy (10). Thus, we need to obtain $\dot{\theta}_{i+1}$ and $\dot{\bar{q}}_{i}$. To this end, using (19) and $\bar{l}_{c i}=\sqrt{x_{c i}^{2}+y_{c i}^{2}}$, we obtain the following iterative relation between $\bar{\beta}_{i}$ and $\bar{\beta}_{i+1}$ :

$\bar{\beta}_{i}=h\left(\beta_{i}, \bar{\beta}_{i+1}, \bar{q}_{i+1}\right), i \in \mathbb{N}_{d}, \bar{\beta}_{d}=\beta_{d}, \bar{\beta}_{n}=\beta_{n}$,

where

$$
h(a, b, z)=\sqrt{a^{2}+b^{2}+2 a b \cos z} .
$$

Using (14), (20), and (21), we obtain

$$
\dot{\bar{q}}_{a}=\Psi\left(q_{a}\right) \dot{q}_{a}, \quad \Psi\left(q_{a}\right)=\left[\begin{array}{cc}
\Psi_{a}\left(q_{a a}\right) & 0 \\
0 & \Psi_{b}\left(q_{a b}\right)
\end{array}\right],
$$

where $\Psi_{a}\left(q_{a a}\right)$ and $\Psi_{b}\left(q_{a b}\right)$ are the following upper triangular matrices:

$$
\Psi_{a}\left(q_{a a}\right)=\left[\begin{array}{cccc}
1 & \psi_{12} & \ldots & \psi_{1(d-1)} \\
0 & 1 & \ldots & \psi_{2(d-1)} \\
\vdots & \vdots & \vdots & \vdots \\
0 & 0 & \ldots & \psi_{(d-2)(d-1)} \\
0 & 0 & \ldots & 1
\end{array}\right],
$$

$$
\Psi_{b}\left(q_{a b}\right)=\left[\begin{array}{cccc}
1 & \psi_{(d+1)(d+2)} & \ldots & \psi_{(d+1) n} \\
0 & 1 & \ldots & \psi_{(d+2) n} \\
\vdots & \vdots & \vdots & \vdots \\
0 & 0 & \ldots & \psi_{(n-1) n} \\
0 & 0 & \ldots & 1
\end{array}\right]
$$


where $\psi_{i j}$ can be obtained directly and is omitted for brevity.

In summary, the transformation $T$ in (13) is given by (16) and (23).

\section{SWING-UP CONTROLLER FOR $n$-LINK ROBOT}

We take the following Lyapunov function candidate:

$$
V=\frac{1}{2}\left(E-E_{r}\right)^{2}+\frac{1}{2} k_{D} \dot{q}_{a}^{\mathrm{T}} \dot{q}_{a}+\frac{1}{2} k_{P} \bar{q}_{a}^{\mathrm{T}} \bar{q}_{a} .
$$

Instead of $q_{a}$ in $V_{A}$, we use $\bar{q}_{a}$ in $V$. Obviously, from (16), we know that $\lim _{t \rightarrow \infty} V=0$ is equivalent to (8).

Taking the time-derivative of $V$ along the trajectories of (1), and using $\dot{E}=\dot{q}^{\mathrm{T}} B \tau=\dot{q}_{a} \tau$ and (23), we obtain

$$
\dot{V}=\dot{q}_{a}^{\mathrm{T}}\left(\left(E-E_{r}\right) \tau+k_{D} \ddot{q}_{a}+k_{P} \Psi^{\mathrm{T}} \bar{q}_{a}\right) .
$$

If we can choose $\tau$ such that

$$
\left(E-E_{r}\right) \tau+k_{D} \ddot{q}_{a}+k_{P} \Psi^{\mathrm{T}} \bar{q}_{a}=-k_{V} \dot{q}_{a}
$$

for some constant $k_{V}>0$, then we have

$$
\dot{V}=-k_{V} \dot{q}_{a}^{\mathrm{T}} \dot{q}_{a} \leq 0 .
$$

We discuss under what condition (27) is solvable with respect to $\tau$ for any $(q, \dot{q})$. From (1), we obtain

$$
\ddot{q}_{a}=B^{\mathrm{T}} \ddot{q}=B^{\mathrm{T}} M^{-1}(B \tau-H-G) .
$$

Substituting (29) into (27) yields

$$
\Lambda(q, \dot{q}) \tau=k_{D} B^{\mathrm{T}} M^{-1}(H+G)-k_{V} \dot{q}_{a}-k_{P} \Psi^{\mathrm{T}} \bar{q}_{a},
$$

where

$$
\Lambda(q, \dot{q})=\left(E(q, \dot{q})-E_{r}\right) I_{n-1}+k_{D} B^{\mathrm{T}} M^{-1} B .
$$

Therefore, when

$$
\operatorname{det} \Lambda(q, \dot{q}) \neq 0, \quad \text { for } \forall q, \forall \dot{q},
$$

we obtain

$$
\tau=\Lambda^{-1}\left(k_{D} B^{\mathrm{T}} M^{-1}(H+G)-k_{V} \dot{q}_{a}-k_{P} \Psi^{\mathrm{T}} \bar{q}_{a}\right) .
$$

We are ready to present the following theorem.

Theorem 1: Consider the robot in (1). Suppose that $k_{D}>$ $0, k_{P}>0$, and $k_{V}>0$. Then the controller (33) has no singular points for any $(q, \dot{q})$ if and only if

$$
\left.k_{D}>k_{D m}=\max _{q_{2}, \ldots, q_{n}}\left\{\left(E_{r}+\mu\right)\right) \lambda_{\max }\left(\left(B^{\mathrm{T}} M^{-1} B\right)^{-1}\right)\right\},
$$

where $\lambda_{\max }(A)$ denotes the maximal eigenvalue of $A>0$,

$$
\mu=\left(\sum_{i=1}^{n} \beta_{i}^{2}+2 \sum_{i=1}^{n-1} \sum_{j>i}^{n} \beta_{i} \beta_{j} \cos \sum_{k=i+1}^{j} q_{k}\right)^{1 / 2} .
$$

In this case, $\lim _{t \rightarrow \infty} V=V^{*}, \lim _{t \rightarrow \infty} E=E^{*}, \lim _{t \rightarrow \infty} q_{a}$ $=q_{a}^{*}, \lim _{t \rightarrow \infty} \bar{q}_{a}=\bar{q}_{a}^{*}$, where $V^{*}, E^{*}, q_{a}^{*}$, and $\bar{q}_{a}^{*}$ are some constants. Moreover, as $t \rightarrow \infty$, every closed-loop solution, $(q(t), \dot{q}(t))$, approaches the invariant set

$$
W=\left\{(q, \dot{q}) \mid \dot{q}_{d}^{2}=\frac{2\left(E^{*}-P\left(q_{1}, q_{a}^{*}\right)\right)}{\left.M_{d d}(q)\right|_{q_{a}=q_{a}^{*}}} ; q_{a} \equiv q_{a}^{*}\right\},
$$

where $M_{d d}$ is the $(d, d)$ element of $M$.

\section{MOTION ANALYSIS OF $n$-LINK ROBOT}

We now characterize the invariant set $W$ in (36) by analyzing the convergent value, $V^{*}$, of the Lyapunov function $V$ in (26). Since $\lim _{t \rightarrow \infty} V=0$ is equivalent to (8), we separately analyze two cases of $V^{*}=0$ and $V^{*} \neq 0$.

Case 1: $V^{*}=0$

From (26) and (16), we have $E^{*}=E_{r}$ and $\bar{q}_{a}^{*}=q_{a}^{*}=0$. Thus, from (36), we obtain

$$
\dot{q}_{d}^{2}=\frac{2 E_{r}}{\left.M_{d d}(q)\right|_{q_{a}=0}}\left(1-\cos q_{d}\right) .
$$

Therefore, as $t \rightarrow \infty$, the closed-loop solution $(q(t), \dot{q}(t))$ approaches the invariant set

$$
W_{r}=\left\{(q, \dot{q}) \mid\left(q_{d}, \dot{q}_{d}\right) \text { satisfies (37); } q_{a} \equiv 0\right\} .
$$

Since (37) is a homoclinic orbit ([10], p.44) with the equilibrium point $\left(q_{d}, \dot{q}_{d}\right)=(0,0),\left(q_{d}(t), \dot{q}_{d}(t)\right)$ will have $(0,0)$ as an $\omega$-limit point; that is, there exists a sequence of times $t_{n}(n=1, \ldots, \infty)$ such that $t_{n} \rightarrow \infty$ as $n \rightarrow \infty$ for which $\lim _{n \rightarrow \infty}\left(q_{d}\left(t_{n}\right), \dot{q}_{d}\left(t_{n}\right)\right)=(0,0)$. Hence, the robot can enter any arbitrarily small neighborhood of the upright equilibrium point as $t \rightarrow \infty$.

Case 2: $V^{*} \neq 0$

Putting $E \equiv E^{*}, q_{a} \equiv q_{a}^{*}$, and $\bar{q}_{a} \equiv \bar{q}_{a}^{*}$ into (27), we show that $E^{*} \neq E_{r}$ and that $\tau$ is a constant vector $\tau^{*}$ satisfying

$$
k_{P} \Psi^{\mathrm{T}}\left(q_{a}^{*}\right) \bar{q}_{a}^{*}+\left(E^{*}-E_{r}\right) \tau^{*}=0, \quad E^{*} \neq E_{r} .
$$

We are ready to present the following lemma.

LEMMA 1: Consider the invariant set $W$ defined in (36). Let $(q(t), \dot{q}(t)) \in W$. If $V^{*} \neq 0$, then in the invariant set $W, q_{d}(t)$ is a constant; that is, $q(t) \equiv q^{*}$ holds.

Putting $q \equiv q^{*}$ and $\tau=\tau^{*}$ into into (1) and (3), we obtain

$$
G_{d}\left(q^{*}\right)=0, \quad \tau^{*}=B^{\mathrm{T}} G\left(q^{*}\right), \quad E^{*}=P\left(q^{*}\right) .
$$

Define the following equilibrium set:

$$
\Omega=\left\{\left(q^{*}, 0\right) \mid q^{*} \text { satisfies (39) and (40) }\right\} .
$$

We are ready to present the following theorem.

Theorem 2: Consider the robot in (1). Suppose that $k_{D}$ satisfies (34), $k_{P}>0$, and $k_{V}>0$. Then under the controller (33), as $t \rightarrow \infty$, the closed-loop solution $(q(t), \dot{q}(t))$ approaches

$$
W=W_{r} \cup \Omega, \text { with } W_{r} \cap \Omega=\emptyset,
$$

where $W_{r}$ is defined in (38), and $\Omega$ is the set of equilibrium points defined in (41).

\section{CLOSED-LOOP EQUILIBRIUM POINTS}

If the set $\Omega$ contains a stable equilibrium point in the sense of the Lyapunov stability, then the robot can not be swung up arbitrarily close to the upright equilibrium point from some neighborhoods close to the stable equilibrium point. 
We will analyze the relationship between $\Omega$ and $k_{P}$. Consider an equilibrium point $\left(q^{*}, 0\right)$ of $\Omega$ in (41). Using $\Psi$ in (23), we can rewrite (39) and (40) as

$$
\begin{aligned}
& G_{d}\left(q^{*}\right)=-\sum_{k=d}^{n} \beta_{i} \sin \sum_{j=1}^{k} q_{j}^{*}=0, \\
& k_{P}\left(\bar{q}_{i}^{*}+\sum_{j=1}^{i-1} \psi_{j i}\left(q_{a a}^{*}\right) \bar{q}_{j}^{*}\right)+\left(P\left(q^{*}\right)-E_{r}\right) \tau_{i}^{*}=0, \\
& \quad \text { for } 1 \leq i \leq d-1, \\
& k_{P}\left(\bar{q}_{i}^{*}+\sum_{j=d+1}^{i-1} \psi_{j i}\left(q_{a b}^{*}\right) \bar{q}_{j}^{*}\right)+\left(P\left(q^{*}\right)-E_{r}\right) \tau_{i}^{*}=0, \\
& P\left(q^{*}\right) \neq E_{r} . \\
& \quad \text { for } d+1 \leq i \leq n,
\end{aligned}
$$

Note that for any given $k_{P}$ the set $\Omega$ contains at least one equilibrium point

$$
\Omega_{0}=\left\{\left(q^{*}, 0\right) \mid q_{a}=0, q_{d}=-\pi\right\},
$$

where links 1 to $d-1$ are in the upright position and links $d$ to $n$ are in the downward position. We investigate how to provide conditions on $k_{P}$ that ensure that the set $\Omega$ does not contain any other equilibrium point. With the two series of VCLs, we show that (43) and (44) can be simplified to equations similar to those for the Pendubot, and (43) and (45) can be simplified to equations similar to those for the Acrobot.

Define $E_{r f}=\sum_{j=1}^{d-1} \beta_{j}$ and $E_{r s}=\sum_{j=d}^{n} \beta_{j}$. One of the main results of this paper is the following theorem.

Theorem 3: Consider the robot in (1). Suppose that $k_{D}$ satisfies (34), and $k_{V}>0$. If $k_{P}$ satisfies

$$
k_{P}>k_{P m}=\max _{1 \leq i \leq n, i \neq d} k_{m i},
$$

where

$$
k_{m 1}=\sup _{\pi \leq w \leq 2 \pi} \frac{E_{r f}\left(2 E_{r s}+E_{r f}(1-\cos w)\right)(-\sin w)}{w},
$$$$
k_{m i}=\left\{\begin{array}{l}
\frac{2}{\pi}\left(\sum_{j=i}^{d-1} \beta_{j}\right)\left(\sum_{j=i}^{n} \beta_{j}\right), \text { if } \beta_{i} \geq\left(\sum_{j=i}^{d-1} \beta_{j+1}\right) \\
\frac{\beta_{i-1}}{\pi}\left(2 E_{r s}+\sum_{j=i-1}^{d-1} \beta_{j}\right), \text { if } \beta_{i}<\left(\sum_{j=i}^{d-1} \beta_{j+1}\right)
\end{array}\right.
$$

for $2 \leq i \leq d-2$ and

$$
\begin{aligned}
k_{m(d-1)} & =\frac{2}{\pi} \min \left(\beta_{d-1}, \beta_{d-2}\right)\left(\min \left(\beta_{d-1}, \beta_{d-2}\right)+E_{r s}\right), \\
k_{m i} & =2 E_{r s} \beta_{i-1}\left(\sum_{j=i}^{n} \beta_{j}\right) /\left(\sum_{j=i-1}^{n} \beta_{j}\right)
\end{aligned}
$$

for $d+1 \leq i \leq n$, then under the controller (33),

1) $\Omega$ in (41) contains only the equilibrium point $\Omega_{0}$ described in (47);

2) $\Omega_{0}$ is unstable in the closed-loop system;
3) the closed-loop solution $(q(t), \dot{q}(t))$ approaches

$$
W=W_{r} \cup \Omega_{0},
$$

as $t \rightarrow \infty$, where $W_{r}$ is defined in (38).

\section{SIMULATION RESULTS}

We verified the theoretical results via a numerical simulation investigation to a 4-link robot with the parameters in Table III. We took $g=9.81 \mathrm{~m} / \mathrm{s}^{2}$.

TABLE III

PARAMETERS OF 4-LINK ROBOT.

\begin{tabular}{ccccc}
\hline Link $i$ & Link 1 & Link 2 & Link 3 & Link 4 \\
\hline$m_{i}[\mathrm{~kg}]$ & 0.20 & 0.20 & 0.10 & 0.10 \\
\hline$l_{i}[\mathrm{~m}]$ & 0.30 & 0.30 & 0.20 & 0.20 \\
\hline$l_{c i}[\mathrm{~m}]$ & $l_{1} / 2$ & $l_{2} / 2$ & $l_{3} / 2$ & $l_{4} / 2$ \\
\hline$J_{i}\left[\mathrm{~kg} \cdot \mathrm{m}^{2}\right]$ & $m_{1} l_{1}^{2} / 12$ & $m_{2} l_{2}^{2} / 12$ & $m_{3} l_{3}^{2} / 12$ & $m_{4} l_{4}^{2} / 12$ \\
\hline
\end{tabular}

For this robot, we showed the numerical results of conditions on control parameters $k_{D}>k_{D m}$ and $k_{P}>k_{P m}$ in Table IV for four cases of $d=1, \ldots$, and $d=4$. We obtained the simulation results of a successful swingup control achieved by the controller (33) with control parameters satisfying conditions given in Table IV. Due to page limitations, we only introduced the results for $d=4$ (we call the robot AAAP robot).

TABLE IV

Conditions on Control Parameters: $k_{D}>k_{D m}$ AND $k_{P}>k_{P m}$

\begin{tabular}{ccccccc}
\hline$d$ & $k_{D m}$ & $k_{m 1}$ & $k_{m 2}$ & $k_{m 3}$ & $k_{m 4}$ & $k_{P m}$ \\
\hline 1 & 0.17 & NA & 3.75 & 1.49 & 0.40 & 3.75 \\
\hline 2 & 0.21 & 1.45 & NA & 0.69 & 0.19 & 1.45 \\
\hline 3 & 0.73 & 2.11 & 0.72 & NA & 0.06 & 2.11 \\
\hline 4 & 1.13 & 2.31 & 0.95 & 0.07 & NA & 2.31 \\
\hline
\end{tabular}

For this AAAP robot, according to Theorem 3 and Table IV, the controller (33) with parameters satisfying $k_{D}>1.13$, $k_{P}>2.31$, and $k_{V}>0$ drives it from any initial state to any arbitrary small neighborhood of the upright equilibrium point or the up-up-up-down equilibrium point. For the initial con-

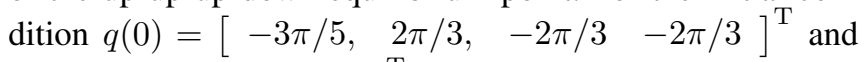
$\dot{q}(0)=\left[\begin{array}{llll}0, & 0,0,0\end{array}\right]^{\mathrm{T}}$, Figs. 3-5 show the simulation results of the controller (33) with $k_{D}=1.17, k_{P}=2.64$, and $k_{V}=5.25$.

Fig. 3 shows that $V$ and $E-E_{r}$ converge to 0. From Fig. 4 , we know that $q_{1}, q_{2}$, and $q_{3}$ converge to 0 , while $q_{4}$ swings closer and closer to 0 after several swings. Finally, the time responses of input torques $\tau_{1}, \tau_{2}$, and $\tau_{3}$ are presented in Fig. 5.

\section{CONCLUSIONS}

In this paper, we aimed to swing up an $n$-link planar robot in a vertical plane with a single passive joint to any arbitrarily small neighborhood of the upright equilibrium point. First, we addressed the problem of how to iteratively devise two sequences of virtual composite links separated by the passive joint as a basis for designing a coordinate transformation 

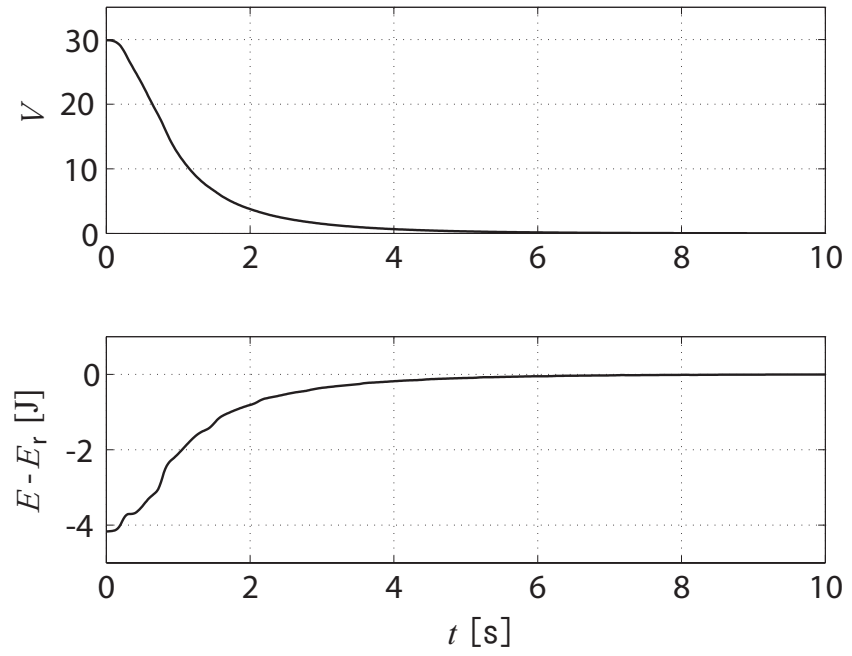

Fig. 3. Time responses of $V$ and $E-E_{r}$.

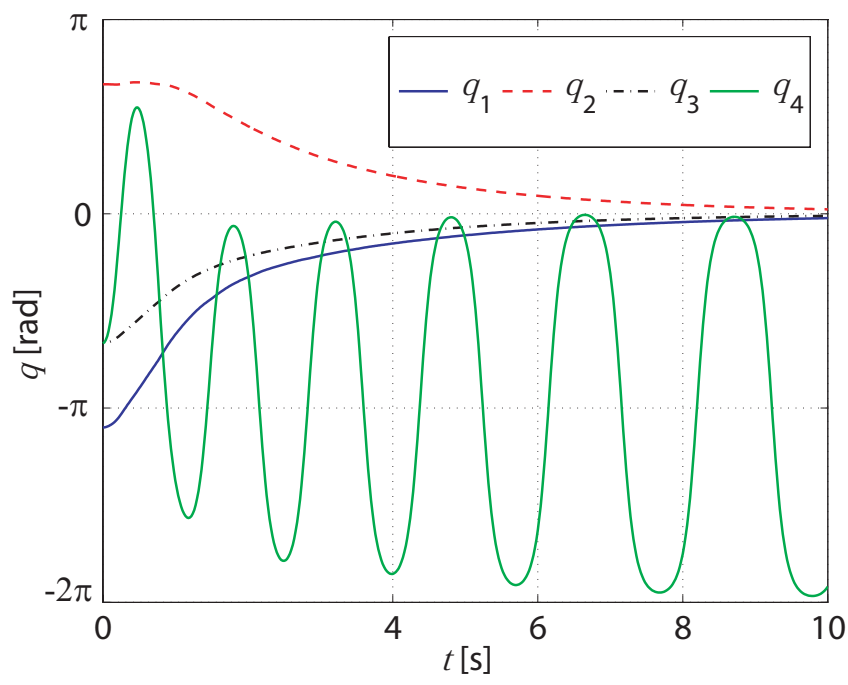

Fig. 4. Time responses of $q_{1}, q_{2}, q_{3}$, and $q_{4}$.

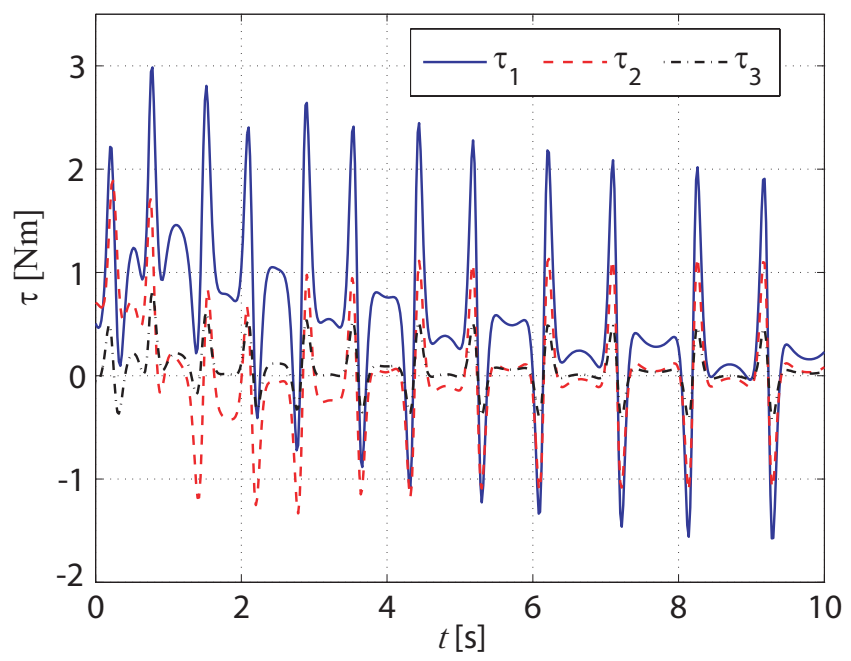

Fig. 5. Time responses of $\tau_{1}, \tau_{2}$, and $\tau_{3}$. on the angles of all active joints. Second, we constructed a Lyapunov function based on the transformation and used it to design a swing-up controller. Third, we analyzed the global analysis of the motion of the robot governed by the devised controller and established conditions on the control parameters that ensure attainment of the swing-up control objective. In fact, we showed that starting from any initial state, the state of the robot will eventually approach either any arbitrarily small neighborhood of the upright equilibrium point, or a certain equilibrium point belonging to the set $\Omega$ in (41). Then, using virtually composite links, we attained conditions on the control parameter $k_{P}$ that ensure that the set $\Omega$ contains only one unstable equilibrium point, about which the robot cannot remain practically. Finally, we validated the theoretical results by means of simulations on a 4-link robot with any one of the joints being a passive joint.

An interesting and challenging future subject is to study an underactuated robot with two or more passive joints.

\section{REFERENCES}

[1] H. Arai, K. Tanie, and N. Shiroma, "Nonholonomic control of a three-DOF planar underactuated manipulator," IEEE Transactions on Robotics and Automation, vol. 14, no. 5, pp. 681-695, 1998.

[2] K. J. Åström and K. Furuta, "Swinging up a pendulum by energy control," Automatica, vol. 36, no. 2, pp. 287-295, 2000.

[3] A. De Luca and G. Oriolo, "Trajectory planning and control for planar robots with passive last joint," International Journal of Robotics Research, vol. 21, no. 5-6, pp. 575-590, 2002.

[4] I. Fantoni, R. Lozano, and M. W. Spong, "Energy based control of the Pendubot," IEEE Transactions on Automatic Control, vol. 45, no. 4, pp. 725-729, 2000.

[5] H. G. Gonzalez-Hernandez, J. Alvarez, and J. Alvarez-Gallegos, "Experimental analysis and control of a chaotic pendubot," International Journal of Robotics Research, vol. 23, no. 9, pp. 891-902, 2004.

[6] J. W. Grizzle, C. H. Moog, and C. Chevallereau, "Nonlinear control of mechanical systems with an unactuated cyclic variable," IEEE Transactions on Automatic Control, vol. 50, no. 5, pp. 559-575, 2005.

[7] O. Kolesnichenko and A. S. Shiriaev, "Partial stabilization of underactuated Euler-Lagrange systems via a class of feedback transformations," Systems \& Control Letters, vol. 45, no. 2, pp. 121-132, 2002.

[8] X. Ma and C. Y. Su, "A new fuzzy approach for swing up control of Pendubot," Proceedings of American Control Conference, pp. 10011006, 2002.

[9] R. Ortega, M. W. Spong, F. Gomez-Estern, and G. Blankenstein, "Stabilization of a class of underactuated mechanical systems via interconnection and damping assignment," IEEE Transactions on Automatic Control, vol. 47, no. 8, pp. 1218-1233, 2002.

[10] S. Sastry, Nonlinear Systems: Analysis, Stability, and Control. Springer, New York, 1999.

[11] M. W. Spong, "The control of underactuated mechanical system," Plenary Address at the First International Conference on Mechatronics, Mexico City, pp. 1-21, 1994.

[12] M. W. Spong, "The swing up control problem for the Acrobot," IEEE Control Systems Magazine, vol. 15, no. 1, pp. 49-55, 1995.

[13] X. Xin and M. Kaneda, "Analysis of the energy based swing-up control of the Acrobot," International Journal of Robust and Nonlinear Control, vol. 17, no. 10, pp. 1503-1524, 2007.

[14] X. Xin and M. Kaneda, "Swing-up control for a 3-DOF gymnastic robot with passive first joint: Design and analysis," IEEE Transactions on Robotics and Automation, vol. 23, no. 6, pp. 1277-1284, 2007.

[15] X. Xin, M. Kaneda, T. Yamasaki, and J. H. She, "Swing-up control based on virtually composite links for an $n$-link underactuated robot with passive first joint," Proceedings of 2008 IFAC World Congress, Seoul, 2008. 